\title{
Satisfactory use of high flow nasal cannula in a patient with acute pulmonary embolism
}

\begin{abstract}
High Flow Nasal Cannula (HFNC) oxygen therapy is a recent technique that delivers a high flow of heated and humidified gas to the patient. ${ }^{1}$ Compared to noninvasive ventilation (NIV), HFNC has been proved to be an effective alternative treatment for acute respiratory failure. HFNC also has a significant number of physiological advantages compared with other commonly used oxygen-based therapies, including PEEP, reduced anatomical dead space, constant $\mathrm{FiO}_{2}$ and it is better tolerated than NIV. ${ }^{2}$ After a thorough bibliographical research, only one article was found assessing the benefits of using HFNC in patients with acute pulmonary embolism. ${ }^{3}$ We present the case of an 86-year-old patient with acute pulmonary embolism and desaturation refractory to noninvasive ventilation that was successfully treated with high flow therapy.
\end{abstract}

Keywords: high flow nasal cannula, noninvasive ventilation, acute pulmonary embolism, chest pain
Volume 5 Issue 6 - 2018

Diurbis Velasco-Álvarez,' Javier Pozas Pérez, Salvador Díaz-Lobato'

'Department of Pneumology, Hospital Universitario Ramón y Cajal, Spain

${ }^{2}$ Department of Medical Oncology, Hospital Universitario

Ramón y Cajal, Spain

\begin{abstract}
Correspondence: Diurbis Velasco Álvarez, Servicio de Neumología, Hospital Universitario Ramón y Cajal, Ctra. de Colmenar Viejo Km 9, I00, 28045 Madrid, Spain, Tel +34 9l 336 80 00, Fax +3491 33680 90, Email diurbisvelasco@gmail.com
\end{abstract}

Received: November 2I, 2018 | Published: December 28, 2018

\section{Introduction}

High Flow Nasal Cannula (HFNC) oxygen therapy is a recent technique that delivers a high flow of heated and humidified gas to the patient. ${ }^{1}$ Compared to noninvasive ventilation (NIV), HFNC has been proved to be an effective alternative treatment for acute respiratory failure. HFNC also has a significant number of physiological advantages compared with other commonly used oxygen-based therapies, including PEEP, reduced anatomical dead space, constant $\mathrm{FiO}_{2}$ and it is better tolerated than NIV. ${ }^{2}$ After a thorough bibliographical research, only one article was found assessing the benefits of using HFNC in patients with acute pulmonary embolism. ${ }^{3}$ We present the case of an 86-year-old patient with acute pulmonary embolism and desaturation refractory to noninvasive ventilation that was successfully treated with high flow therapy.

\section{Case report}

An 86-year-old woman who suffers from a poorly controlled permanent atrial fibrillation treated with Rivaroxaban. On June 2018, the patient presented to the Emergency Department referring a sudden onset dyspnea and agitation, without chest pain or syncope. The patient has a history of severe pulmonary embolism in 2004. Despite being anticoagulated with Rivaroxaban, the adherence to treatment was not optimal and the patient had not complied with it since 4 days prior to her admission into the Emergency ward.

At her arrival, she presented tachycardia (120 beats per minute), normal blood pressure $(132 / 90 \mathrm{mmHg})$ and the hemoglobin saturation of oxygen was $76 \%$. When it came to physical examination, the patient has normal breath sounds, signs of chronic venous deficiency but no clinical data suggestive of deep vein thrombosis. The laboratory results showed troponin: $0.3 \mathrm{ng} / \mathrm{mL}$; brain natriuretic peptide: $1336 \mathrm{pg} /$ $\mathrm{mL}$; leucocytes: 14000 with neutrophilia; creatinine: 1.58 . Arterial gasometry revealed $\mathrm{pH}: 7.41 ; \mathrm{pCO}_{2}: 31.0 \mathrm{mmHg} ; \mathrm{pO}^{2}: 34.0 \mathrm{mmHg}$; $\mathrm{HCO}_{3}: 17.9 \mathrm{mM} / \mathrm{L}$.

Wells score indicated a high probability of pulmonary embolism, hence anticoagulation with low molecular weight heparin at therapeutic dose was initiated. CT pulmonary angiography revealed filling defects affecting the right main pulmonary artery, right lobar and segmental arteries, left lobar artery and lingular artery, all this findings being compatible with acute pulmonary embolism (Figure 1). Diuretic treatment with Furosemide was optimized and despite all the measures, the dyspnea persisted and oxygen saturation was still low. Therefore, noninvasive ventilation was started. After one hour of therapy, the oxygen saturation values were around $83 \%$, so NIV was substituted by $\mathrm{HFNC}$, reaching a $\mathrm{SatO}_{2}$ of $94 \%$ in the following minutes.

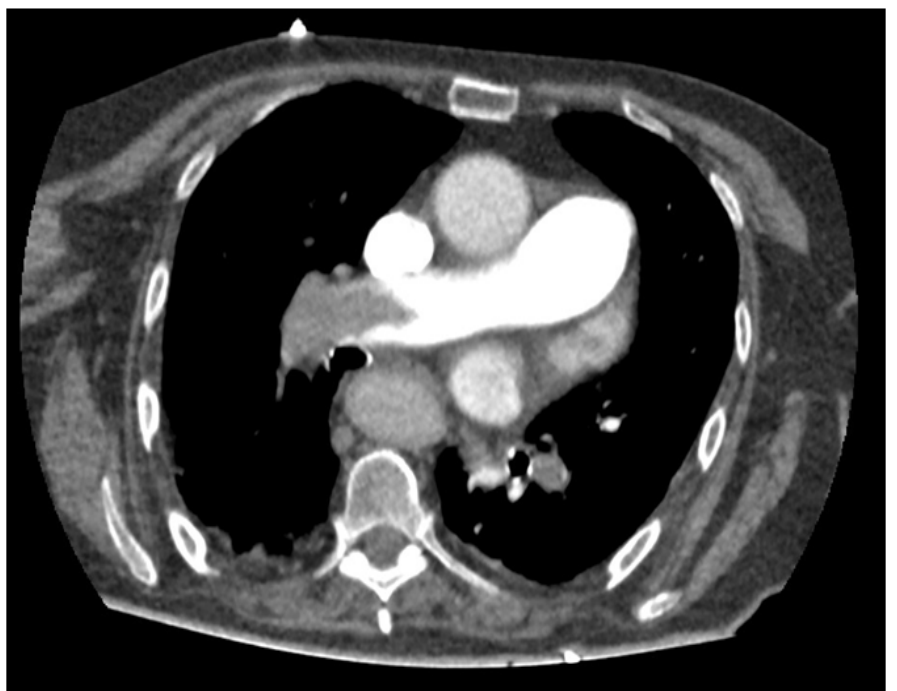

Figure I CT pulmonary angiography showing acute pulmonary embolism.

The patient remained clinically stable during the following days, allowing a progressive reduction of the flow. After 5 days in the Pneumology ward, weaning of HFNC was possible, maintaining good oxygen saturation values and hospital discharge was decided. 


\section{Discussion}

HFNC has several advantages such as providing higher flow rates, which creates a positive pressure effect and reduces the anatomic dead space; delivering a predictable and constant $\mathrm{FiO} 2,{ }^{4}$ increasing the partial arterial pressure of oxygen $(\mathrm{PaO} 2) / \mathrm{FIO} 2$ ratio, which reduces the entrainment of room air and the dilution of oxygen; providing heated and humidified gas that is inhaled and can improve mucociliary motion and sputum clearance; reducing upper airway resistance and work of breathing; and improving thoraco-abdominal synchrony. Based on the above advantages, several studies found that HFNC could improve comfort level, increase oxygenation and decrease the dyspnea score in adult patients.

Even though superiority is still being studied seems that Highflow nasal cannula oxygen therapy (HFNC) is superior to noninvasive ventilation (NIV) and conventional oxygen therapy in hypoxemic patients in terms of mortality and avoidance of intubation. ${ }^{5}$ Noninvasive ventilation in pulmonary embolism increases intrathoracic pressure, which may result in a decrease of right stroke volume and arterial pressure, and this is contrary to therapy objectives in PE.

Only a few cases of pulmonary embolism treated with HFNC have been reported. Messika et al. ${ }^{3}$ analyzed 17 patients with severe PE (determined by profound desaturation under room air, $\mathrm{SpO}_{2} 85 \%$ [7089]) and HFNC administration for acute respiratory failure (defined by the need for at least $6 \mathrm{l} / \mathrm{min}$ of oxygen to achieve $\mathrm{SpO}_{2}>92 \%$ ), concluding that respiratory parameters significantly improved after to 2hours of therapy with HFNC: $\mathrm{SpO}_{2}$ increased from $93 \%$ to $100 \%$, respiratory rate decreased from 29 to 20 cycles/min and hemodynamic parameters did not show significant variations after the initiation of HFNC.
Therefore, this clinical report supports the previous published data, suggesting that HFNC is an effective therapy for patients with desaturation during the acute phase of pulmonary embolism. However, the authors emphasize the need for more randomized clinical trials to assess the effectiveness of HFNC in this type of patients.

\section{Acknowledgments}

None.

\section{Conflicts of interest}

The authors declare no conflicts of interest.

\section{References}

1. Frat JP, Coudroy R, Marjanovic N, et al. High-flow nasal oxygen therapy and noninvasive ventilation in the management of acute hypoxemic respiratory failure. Ann Transl Med. 2017;5(14):297.

2. Nishimura M. High-Flow Nasal Cannula Oxygen Therapy in Adults: Physiological Benefits, Indication, Clinical Benefits, and Adverse Effects. Respiratory Care. 2016;61(4):529-541.

3. Messika J, Goutorbe P, Hajage D, et al. Severe pulmonary embolism managed with high-flow nasal cannula oxygen therapy. Eur J Emerg Med. 2017;24(3):230-232.

4. Nishimura M. High-flow nasal cannula oxygen therapy in adults. $J$ Intensive Care. 2015;3(1):15.

5. Zhao H, Wang H, Sun F, Lyu S and An Y. High-flow nasal cannula oxygen therapy is superior to conventional oxygen therapy but not to noninvasive mechanical ventilation on intubation rate: a systematic review and meta-analysis. Critical Care. 2017;21(1):184. 